\title{
Remote monitoring and control system for Communication Stations based on JavaFX and Web Service
}

\author{
Yang Yue-xin ${ }^{1, a}$, Sun Lian-shan ${ }^{1, b}$ \\ ${ }^{1}$ College of Electrical and Information Engineering , Shaanxi University of Science \& Technology, \\ Xi'an 710021,China \\ ayangyuexin2005@126.com, bsunlianshan@sust.edu.cn
}

Keywords: JavaFX; Web Service; Communication stations; Monitoring and control system; ARM

\begin{abstract}
The paper proposed a remote monitoring and control system based on JavaFX and Web Service .In the system, communication stations were disbursed over a wide area. In a cell site, different kinds of sensors communicate with station program based on ARM. Cell sites uploaded the information of alarms and videos to the server. Server provided remote methods to the remote client through the Web Service. Operation of the system shows that, the proposed system has good openness and expansibility.
\end{abstract}

\section{Introduction}

Along with the rapid growth in the communication industry, communication enterprise co nstructed a large number of communication base stations. The communication base stations located on wide distribution, in order to strengthen the management of communication ba se station and improve the communication enterprise communication network maintenance le vel, it is needed to solve the management problems of communication base station.Commun ication enterprise to various independent base station real-time to monitor the situation, acce ss to the base station fire and security alarm information and inform the relevant personnel to deal with, so as to realize the communication base stations of unmanned, improve the $s$ afety and reliability of communication equipment. In order to solve the above problem, we design framework for remote base station monitoring system based on the JavaFX and Web Service.

\section{The framework of the System}

The structure of remote monitoring and control system needs to meet the demand of co mmunication base stations. The communication base stations processing program will upload the real-time status of the base stations and alarm informations to the center database ser ver, so that the alarm information can be responsed and dealed, improving the reliability o f communication network.Figure 1 shows the structure of the system.

The System consists of the following three parts:

Monitoring terminal: the base station processing program based on ARM9 and moun ted in the base station within the various types of sensors. The base station processing prog ram based on the ARM9 communicate with the sensors through the RS485 serial, communi cate withe the communication enterprise through local area network . 


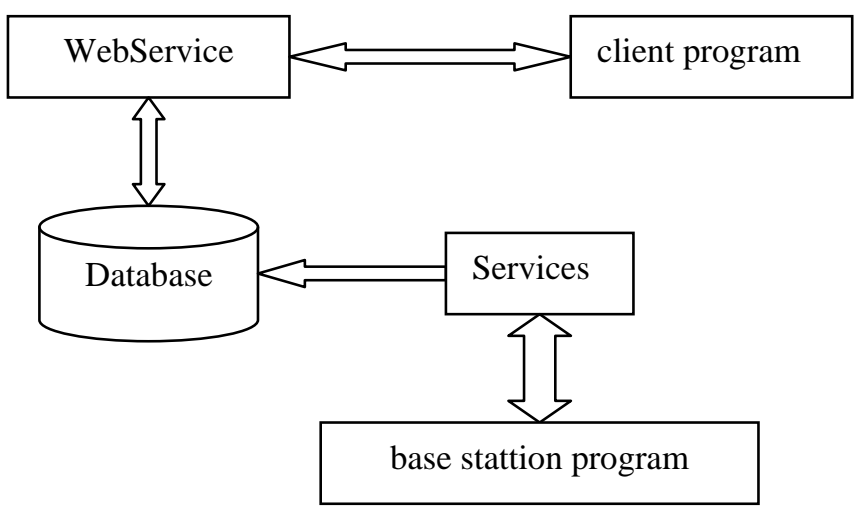

Fig. 1 the structure of the system

Server: located in the computer room, consists of four related services (the alarm service, the video service, the measure service, the data maintenance service) and the web service which provided the remoting method.

Client: located in different departments of communication enterprises, different users use $t$ he client to view real-time remote station real time status, timely access to the base station alarm and video information.

\section{The overall structure of base station}

The communication base station had low power consumption by using the ARM9 proces sor and embedded technology. Embedded processing technology is the trend of development.

The system selected the Samsung Corp's S3C2440A. The kernel is a special industrial cont rol ARM9 chip which has a rich application interface: frequency 533MHz; two USB 2 host port and a USB 2 device port; an 10M Ethernet RJ-45 interface, a serial port 3. Its rich external equipment and the data transmission characteristics are its advantagies. Figure 3 sh ows the structure of the hardware in the base station

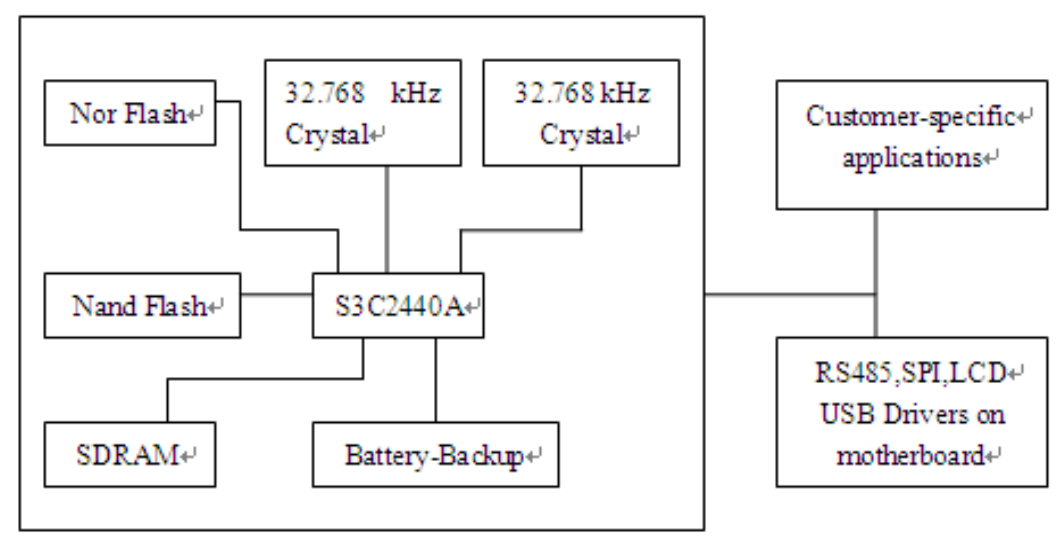

Fig. 2 the structure of the hardware in the base station

\section{The design of server program}

The server program consists of four service and the Web Service which provides the re moting method to the remote monitoring client. The structure of the server program is sho wn in figure 3.

Alarm service: it communicated with the base station through the TCP/IP protocol. It can access the alarm information of the the base station. By using the alarm service the alarm 
information stored in the database.

Video service: it communicated with the base station through the TCP/IP protocol. By $u$ sing the video service the video information stored in the database. At the same time, the video file can be uploaded to the directory for videos in the server.

Measure service: It is used for get the status of all base stations and the current value of the sensors located in one base station through TCP/IP protocol.

Maintenance service: It is used at the idle time of the server. It delete the alarm record and the corresponding video record in order to ensure the server efficiency.

Web Service: It provides the remote base station monitoring client the remote methods, $\mathrm{s}$ uch as the method which gets the video information.

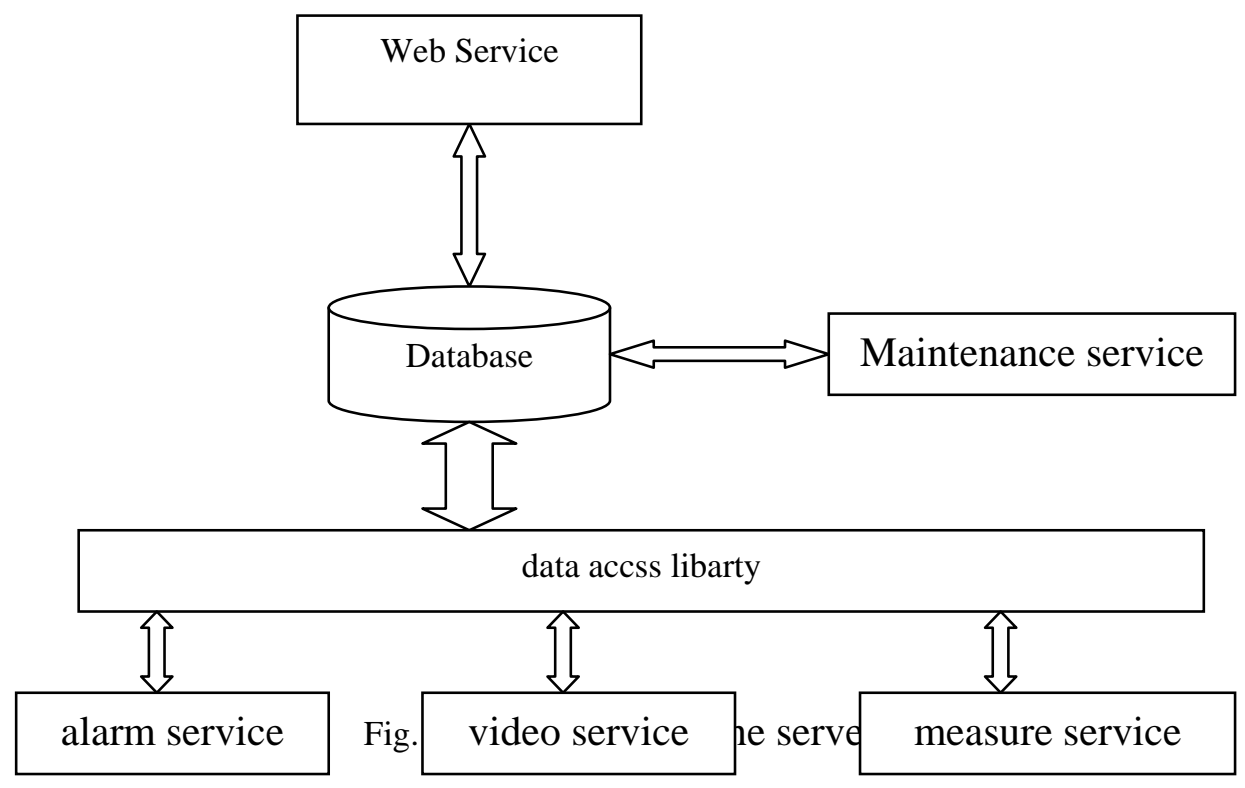

\section{The design of client program}

The client program is coded in JavaFX. The core of the JavaFX is JavaFX script langu age. The JavaFX Script using java swing interface library to create the RIA program. The program can be run on JVM. The JavaFX realize the application by using attributes. The J avaFX Script provides procedural and declarative syntax with the functional language feature s. It can use the java libarry directly. By combining with Hessian the JavaFX can access $t$ he Web Service on the server, JavaFX. In the client program, the JavaFX is used to design user interface.The client progarm has the following modular:

Base stations management modular: adding new base station, delete and modify the base stations.

Alarm modular: search the latest alarms and deal with the alarm.

Video modular: download and Download View: an alarm corresponding video informatio $\mathrm{n}$ and the client local video player to view;

User management modular: adding, deleting, modifing the users.

Report view modular: According to the user's selection dynamically generate the repor $\mathrm{t}$ in a region.

Import and export modular: importing the base station information to the database; expor ting the alarm information to a excel file.

\section{Advantages of the system}

The system has the following advantages:

The alarm information can be uploaded to the central server in time, the client can ti mely show alarm information.

By using the system a wide area can be monitored. 
By using the system a new base station can be added by the client, meeting the needs of the development of enterprises;

\section{Acknowledgements}

This study was supported by the National Natural Science Foundation of China, No 61202019 and Optional project of Shaanxi University of Science and Technology(No.ZX10-31).

\section{Conclusions}

This paper describes the framework of the remote base station monitoring system basedo $\mathrm{n}$ JavaFX and Web Service. Base station monitoring system based on ARM. The base stati on program achieve the sensors timely. By using Web Service, the remote client can get $t$ he alarm information timely. By using the system the communication company can monitor the widely scattered base stations, timely get the status of the base station such as fire ala rm. The system can realize real time and all - weather monitoring. The system can improv e safety of the base stations. With less investment, it greatly improve the communication en terprise's technological level and management level.

\section{References}

[1] ZHAO Wei, PENG Chu-wu, Design of GPRS Based Monitoring System for Comunication Base Station, Computing Technology and Autonmation. (2009) 59-62.

[2] CHEN Zhi-jian,ZHANG Ning-ning,ZHANG Xiong-wen, Settlement monitoring system of pile-group foundation , Journal of Central South University of Technology(English Edition). (2011) 2123-2130.

[3] LIANG Gen, Li Wen, Design of remote monitoring and control system for power plants based on distributed OPC,components linkage and Web Service, Electric Power Automation Equipment. (2011) 134-138.

[4] ZHOU Shao-mei,WANG Hong-lei,LIU Xiao-dong, Application of JSF and JavaFX in RIA Develompment , Computer Applications And Software. (2009) 118-119.

[5] PENG Jian-wei, The Reconstruction of Supervision and Control System for Power and Environment in Mobile Base Stations, Telecom Power Technologies . (2005) 53-54.

[6] DONG Yu-yi, LIU Xin-wei, The Reconstruction of Supervision and Control System for Power and Environment in Mobile Base Stations, Radio Communications Technology. (2012) 73-76.

[7] WANG Xiao-liang, SONG Zhen-guo, AN Yi, A Remote Real-time Data Collection Monitor and Control System Based on GPRS, Instrument Technique and Sensor. (2005) 60-62.

[8] WANG Yu-tian,QIN Rui-feng,LIU Jian-yuan,LI Peng Remote Monitor System for Oil Wells Based on GPRS, Instrument Technique and Sensor. (2009) 45-47.

[9] ZHANG Hai-ning,DU Jun,QI Zhong,JIN Yan-lei, Web publishing system of power security and stability control system, Electric Power Automation Equipment. (2009) 128-131.

[10]ZHU Bo,YAN Chun-ping, Networked Optimal Cutting-stock System Based on Web Service , Journal Of ChongQing Uunversity (Natural Science Edition). (2005) 19-23. 